\title{
Rapid gel-card agglutination assays for serological analysis following SARS-CoV-2 infection in
}

\section{humans.}

Diana Alves, ${ }^{1,2+}$ Rodrigo Curvello, ${ }^{1,2+}$ Edward Henderson, ${ }^{1,2,3 \neq}$ Vidhishri Kesarwani, ${ }^{1,2,3+}$ Julia A. Walker, ${ }^{1,2,3,4:}$ Samuel C. Leguizamon, ${ }^{1,2,5,6 *}$ Heather McLiesh, ${ }^{1,2, *}$ Vikram Singh

Raghuwanshi, ${ }^{1,27}$ Hajar Samadian, ${ }^{1,27}$ Erica M. Wood, ${ }^{7,8}$ Zoe K. McQuilten, ${ }^{7,8}$ Maryza Graham, 9,10,11 Megan Wieringa, ${ }^{9,11}$ Tony M. Korman, ${ }^{9,10,12}$ Timothy F. Scott, ${ }^{1,2,5}$ Mark M. Banaszak Holl, ${ }^{1,2}$ Gil Garnier, ${ }^{* 1,2}$ Simon R Corrie $e^{* 1,2,3,4}$

${ }^{1}$ Department of Chemical Engineering, ARC Centre of Excellence in Convergent BioNano

Science and Technology, Monash University, Clayton, Victoria, 3800, Australia

${ }^{2}$ Bioresource Processing Research Institute of Australia (BioPRIA), Monash University, Clayton, Victoria 3800, Australia

${ }^{3}$ Centre to Impact AMR, Monash University, Clayton, Victoria, 3800, Australia

${ }^{4}$ Monash Institute of Pharmaceutical Sciences, Monash University, Parkville, Victoria, 3052, Australia

${ }^{5}$ Department of Materials Science and Engineering, Monash University, Clayton, Victoria, 3800, Australia 
${ }^{6}$ Department of Chemical Engineering, University of Michigan, Ann Arbor, MI, 48109, USA

${ }^{7}$ Department of Clinical Haematology, Monash Health, Clayton, Victoria, 3168, Australia

${ }^{8}$ Department of Epidemiology and Preventive Medicine, Monash University, Melbourne, Victoria, 3004, Australia

${ }^{9}$ Department of Microbiology, Monash Health, Clayton, Victoria, 3168, Australia

${ }^{10}$ Monash Infectious Diseases, Monash Health, Clayton, Victoria, 3168, Australia

${ }^{11}$ Department of Clinical Sciences, Monash University, Clayton, Victoria, 3168, Australia

${ }^{12}$ Center for Inflammatory Diseases, Department of Medicine, Monash University, Clayton, Victoria, 3800, Australia 


\section{Peptide 1}

FAM-GGG-PLQSYGFQPTNGVGY-GGGCG

$M W: 2487.60 \mathrm{~g} / \mathrm{mol}$
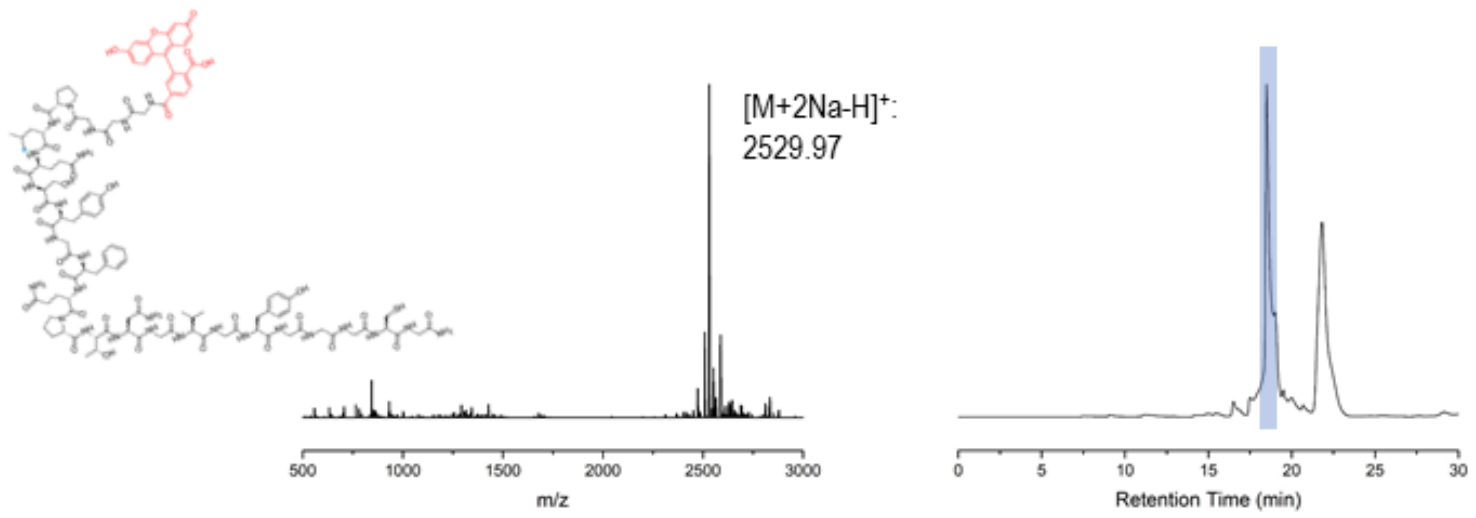

\section{Peptide 2}

FAM-GGG-FSQILPDPSKPSKRSFIE-GGGCG

MW: $2936.21 \mathrm{~g} / \mathrm{mol}$
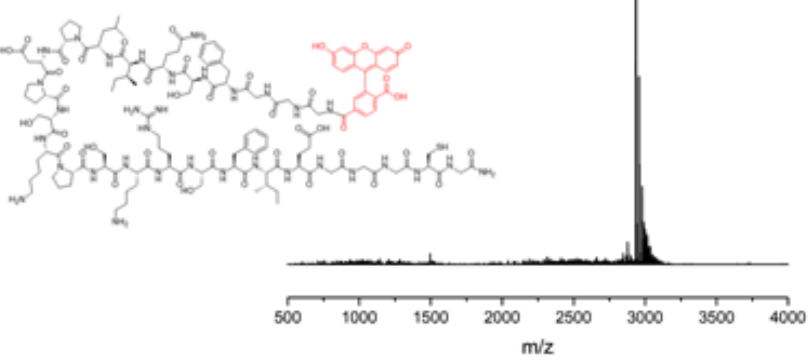

Peptide 5

FAM-GGG-FGAGAALQIPFAMQMAYRFNGI-GGGCG

MW: $3234.64 \mathrm{~g} / \mathrm{mol}$

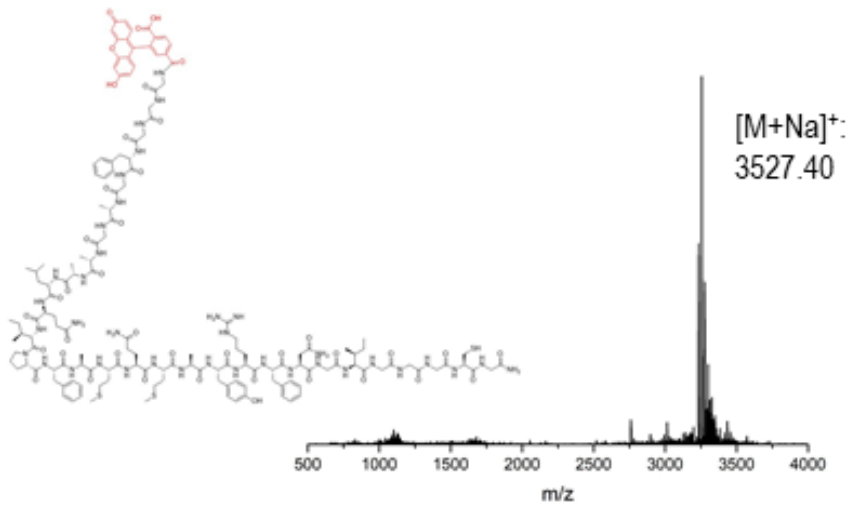

$[\mathrm{M}+\mathrm{H}]^{+}$

2935.09

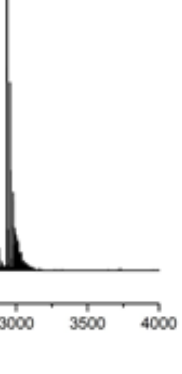

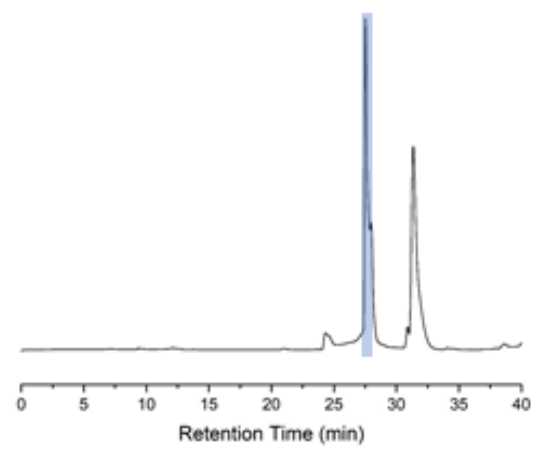

Figure S1: Peptide sequences, expected structures and LC/MS results.

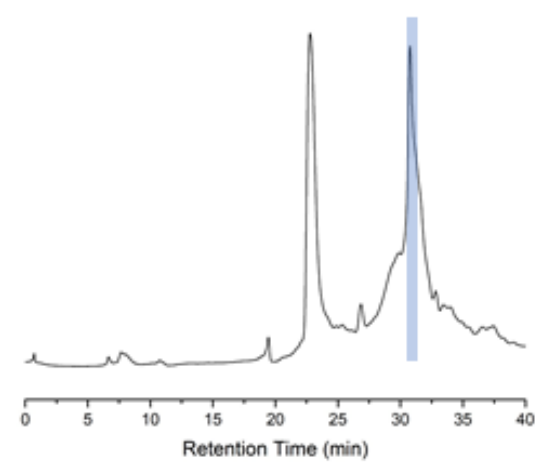




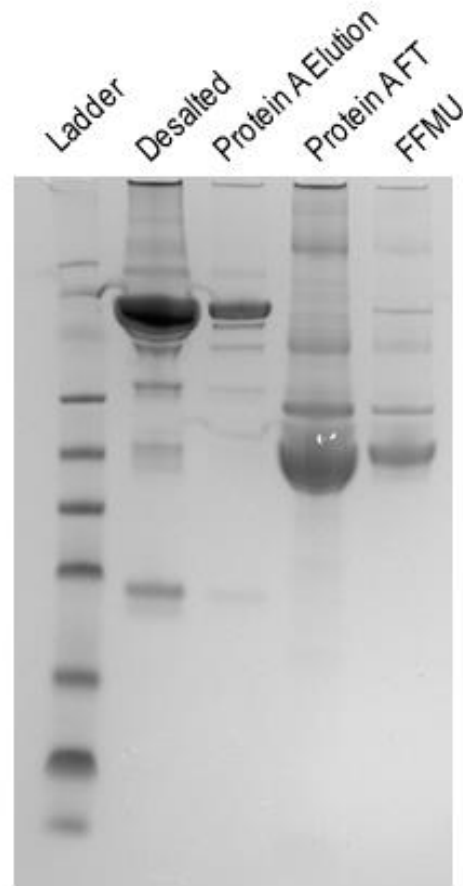

Figure S2: SDS-PAGE gel showing results of Protein A-purification of anti-D-IgG FFMU supernatant to purified and desalted anti-DIgG. "Protein A FT" = flow through from Protein A column; "Protein A Elution" = eluant from Protein A column, "Desalted" = concentrated product after buffer exchange into PBS. 
(A) False positives
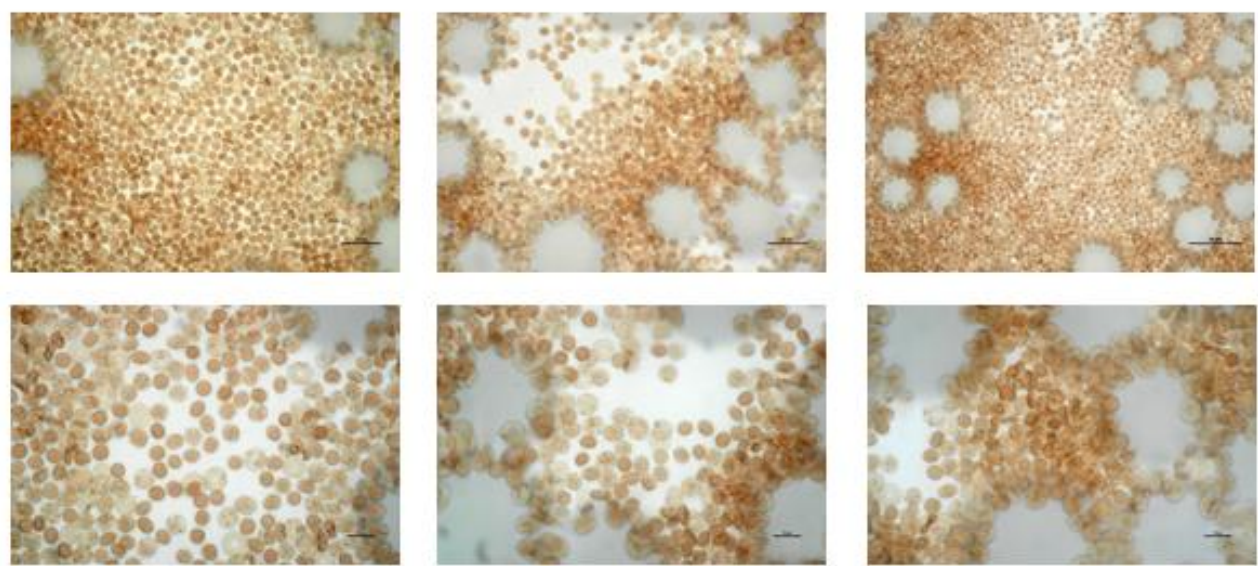

(B) True Positives
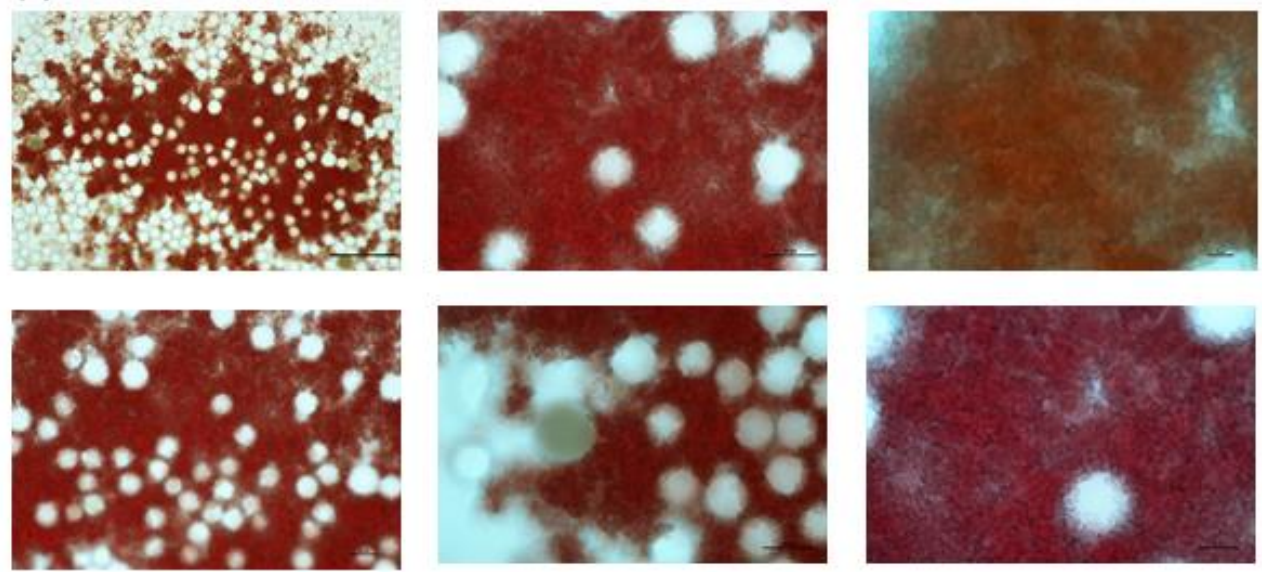

Figure S3: Light microscopy images of RRBCs collected from the top layer of gel cards. (A) False positive gel cards revealed cells that were not agglutinated, and showed some apparent interactions with the gel beads. While retained on top of the gel card, the lack of agglutination confirms the false positive status. This was most often associated with the presence of glycerol in the RRBC stocks, carried over from the bioconjugate solutions. (B) True positive gel cards revealed clear regions of strong agglutination, a 3D network of cells aggregated together in a continuous pattern. 
Table S1: List of clinical samples used in the study

\begin{tabular}{|l|l|l|}
\hline Sample & $\begin{array}{l}\text { Time between PCR and sample } \\
\text { collection (Date of collection) }\end{array}$ & Sample Matrix \\
\hline COVID19-positive (PCR-confirmed) & Serum \\
\hline 1 & 30 & Serum \\
\hline 2 & 34 & Serum \\
\hline 3 & 8 & Serum \\
\hline 4 & 27 & Serum \\
\hline 5 & 28 & Serum \\
\hline COVID19-negative (collected prior to pandemic) \\
\hline 6 & $07 / 19 / 2017$ & Plasma \\
\hline 7 & $01 / 20 / 2014$ & Plasma \\
\hline 8 & $07 / 28 / 2014$ & Serum \\
\hline 9 & $05 / 21 / 2012$ & Serum \\
\hline 10 & $12 / 02 / 2015$ & \\
\hline
\end{tabular}

